\title{
The Balloon-borne Large Aperture Submillimetre Telescope (BLAST) and BLASTPol
}

\author{
Enzo Pascale \\ for the BLAST and BLASTPol collaboration
}

School of Physics \& Astronomy, Cardiff University, 5 The Parade, Cardiff, CF24 3AA, UK

\begin{abstract}
Balloon observations from Antarctica have proven an effective and efficient way to address open Cosmological questions as well as problems in Galactic astronomy. The Balloonborne Large Aperture Submillimetre Telescope (BLAST) is a sub-orbital mapping experiment which uses 270 bolometric detectors to image the sky in three wavebands centred at 250,350 and $500 \mu \mathrm{m}$ with a $1.8 \mathrm{~m}$ telescope. In the years before Herschel launched, BLAST provided data of unprecedented angular and spectral coverage in frequency bands close to the peak of dust emission in star forming regions in our Galaxy, and in galaxies at cosmological distances. More recently, BLASTPol was obtained by reconfiguring the BLAST focal plane as a submillimetric polarimeter to study the role that Galactic magnetic fields have in regulating the processes of star-formation. The first and successful BLASTPol flight from Antarctica in 2010 is followed by a second flight, currently scheduled for the end of 2012 .
\end{abstract}

\section{Introduction}

The Balloon-borne Large Aperture Submillimeter Telescope (BLAST, Pascale et al. 2008) is a sub-orbital surveying experiment designed to study the evolutionary history and processes of star formation in local galaxies (including the Milky Way) and galaxies at cosmological distances. The BLAST continuum camera, which consists of 270 detectors distributed between three arrays, observes simultaneously in broad-band (30\%) spectral-windows at 250,350 and $500 \mu \mathrm{m}$. The $1.8 \mathrm{~m}$ Ritchey-Chrétien telescope provides resolutions of $36^{\prime \prime}, 42^{\prime \prime}$ and $60^{\prime \prime}$ at 250,350 and $500 \mu \mathrm{m}$, respectively. BLAST is a forerunner of the Spectral and Photometric Imaging Receiver (Griffin et al. 2010) on the $3.5 \mathrm{~m}$ Herschel space observatory, and with SPIRE it shared similar focal plane technology and scientific goals.

In the years preceding the launch of Herschel, BLAST made three flights. A $24 \mathrm{hr}$ test flight from Fort Sumner, and two long-duration science flights - from Kiruna, Sweden in 2005, and from Antarctica in 2006. The BLAST cosmological surveys, centered on the South Ecliptic Pole (Valiante et al. 2010) and on the Great Observatories Origins Deep Survey (GOODS-South, see Devlin et al. 2009), were conducted during the 2006 flight, at an altitude of $38 \mathrm{~km}$. The GOODS-South region combines a wide-area map of 8.7 square degrees with a deeper, confusion-limited map of 0.8 square degrees. We refer to these fields as BLAST GOODS-S Wide (BGS-Wide), and BLAST GOODS-S Deep (BGS-Deep) respectively. The BGS-Deep survey is dominated by the point-source confusion arising from the $36^{\prime \prime}-60^{\prime \prime}$ BLAST beams, rather than instrumental noise. The BLAST data, combined with existing photometric measurements taken from the optical to the FIR, and with the available redshift information, have enabled Marsden et al. (2009) and Pascale et al. (2009) to use stacking analysis to estimate the contribution to the Cosmic Infrared Background (CIB) at wavelengths close to its peak. Patanchon 


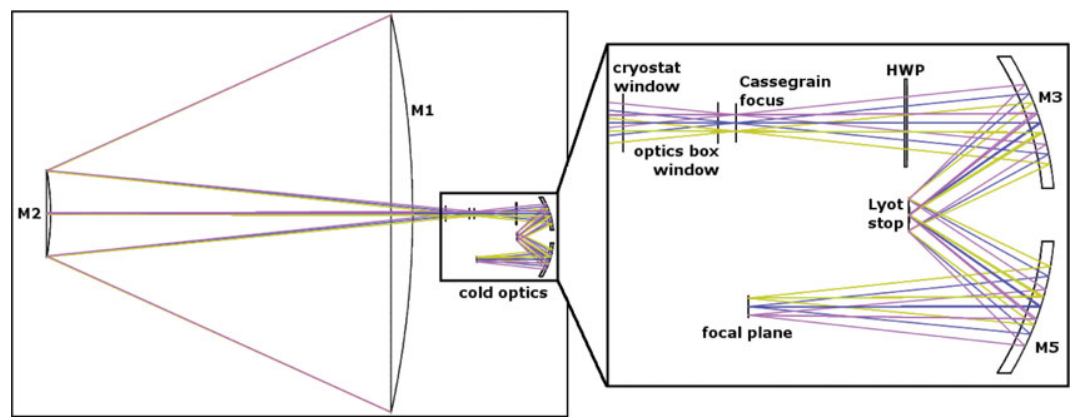

Figure 1. Schematic of the optical layout for the BLAST telescope and receiver shown on the left, with the $1.5 \mathrm{~K}$ optics, located within the cryostat, shown in an expanded view on the right. The image of the sky formed at the input aperture is re-imaged onto the bolometer detector arrays at the focal plane. The M4 mirror serves as a Lyot stop, which defines the illumination of the primary mirror for each element of the bolometer detector arrays. The three wavelength bands are separated by a pair of dichroic beam-splitters (not shown here). The sapphire half-wave plate used in BLASTPol is also shown, mounted $19 \mathrm{~cm}$ behind the focus of the telescope.

et al. (2009) studied the pixel histogram of the Deep and Wide maps to constrain a parametric model of differential source counts. Viero et al. (2009) detected the clustering signal of the submillimetric sources at 250, 350 and $500 \mu \mathrm{m}$. A 50 square degree survey on the Vela Molecular Ridge (Netterfield et al. 2009) was also conducted in 2006, revealing large numbers of starless and pre-stellar cores, which are the earliest stages of starformation.

In May 2009, Herschel was successfully launched and all BLAST targets have been observed from space at the same wavelengths, but with the improved sensitivity and angular resolution of SPIRE. The BLAST multiband photometer, no longer necessary, was re-configured as a polarimeter, resulting in BLASTPol, a enhanced version of BLAST. With the addition of a polarizing grid in front of each of the 270 feed horns and a stepped achromatic Half Wave Plate (AHWP), BLASTPol is now an instrument designed to measure polarized dust emission from star-forming regions. By mapping polarization from dust grains aligned with respect to their local magnetic field, the field orientation (projected on the sky) can be traced. The magnetic-field strength can also be estimated indirectly using the polarization angular dispersion (Chandrasekhar \& Fermi 1953).

During the first flight of BLASTPol in December 2010 from McMurdo, Antarctica, we made sensitive degree-scale maps of several nearby molecular clouds. While the angular resolution $\left(<1.5^{\prime}\right)$ was poorer than planned due to a blocking filter that was melted by the Sun on ascent, our preliminary polarization maps indicate coherent polarization across our target clouds at the few percent level.

This BLASTPol dataset is being used to investigate the role that magnetic fields play in the star-formation process, an important outstanding question in our understanding of how stars form. BLASTPol maps of magnetic fields cover entire Giant Molecular Clouds (GMCs), yet have sufficient resolution to probe fields in dense filamentary sub-structures and molecular cores. The experiment provides a crucial bridge between the large area but coarse resolution polarimetry provided by experiments such as Planck and the high resolution but small area of ALMA data.

A second flight is scheduled to occur in December 2012 from Antarctica to improve on the area, depth and angular resolution obtained during the 2010 flight. 


\section{INSTRUMENT}

A detailed description of the BLAST instrument is given in Pascale et al. (2008) and Marsden et al. (2008). The main features of the optical system are summarized in Fig. 1. The Ritchey-Chrétien telescope has an aluminium primary mirror with a diameter of $1.8 \mathrm{~m}$. The radiation collected is re-imaged by a series of cold $(\sim 1.5 \mathrm{~K})$ reflecting optical elements arranged into an ideal Offner relay inside a long-duration cryostat. This cryostat uses liquid helium and nitrogen and has a hold time of more than 10 days. The telescope's secondary mirror is actuated, so the system can be refocused in-flight. The light is split into three $30 \%$-wide submillimetric bands respectively, centered at 250,350 and $500 \mu \mathrm{m}$. The BLAST focal plane consists of arrays of 149, 88 and 43 detectors at 250, 350 and $500 \mu \mathrm{m}$ respectively. The arrays are cooled to $270 \mathrm{mK}$. Each array element is a silicon nitride micromesh "spiderweb" bolometer (Glenn et al. 1998), coupled to the front optics by a smooth-walled conical feed-horn (Chattopadhyay et al. 2003).

The BLASTPol instrument (Fissel et al. 2010) is a modification of the BLAST telescope that adds linear polarization capabilities. This is achieved by adding a polarizing grid at the mouth of each feed-horn, and a stepped achromatic half-wave plate (AHWP) to modulate the polarization.

The grids are patterned to alternate the polarization angle sampled by $90^{\circ}$ from hornto-horn and thus bolometer-to-bolometer along the scan direction. This arrangement has proved effective in rejecting $1 / f$ noise correlated among detectors in an array (array common modes). BLASTPol scans so that a source on the sky passes along a row of detectors, and thus the time required to measure one Stokes parameter $(Q$ or $U$ ) is just equal to the separation between bolometers divided by the scan speed. For the $250 \mu \mathrm{m}$ detector array where the bolometers are separated by $45^{\prime \prime}$, and assuming a typical scan speed of $0.05^{\circ} / \mathrm{s}$, this time is $0.25 \mathrm{~s}$. This timescale is short compared to the characteristic low frequency $(1 / f)$ noise knee for the detectors at $0.035 \mathrm{mHz}$ (Pascale et al. 2008).

The use of a cryogenic, stepped AHWP (Moncelsi et al. 2012) allows modulation of the Stokes parameters $Q$ and $U$ such that each detector measures $I, Q$, and $U$ multiple times in each sky direction. A total of four AHWP position angles are used (at 0, 22.5, 45 and $67.5^{\circ}$ ), stepped at the end of the telescope's raster-scan on a given target. This mitigates the effect of unbalanced gains between adjacent detectors which would result in a large bias on the estimated $Q$ and $U$ if only detector differences are used to estimate the Stokes parameters.

Each astronomical target was observed with BLAST (or BLASTPol) in a slow rasterscan mode. Slow scanning is preferable to a mechanical chopper for mapping large regions of the sky. The telescope is scanned in azimuth at a constant velocity of $\sim 0.05^{\circ} / \mathrm{s}$. At the end of each azimuthal scan, the elevation is stepped by $1 / 3$ of the array's $7^{\prime}$ field of view $(\mathrm{FOV})$ in elevation (the array FOV is $14^{\prime} \times 7^{\prime}$ ).

\section{BLAST Observations}

The BLAST point source catalog (Devlin et al. 2009) lists hundreds of sources detected in the BGS-Wide and BGS-Deep regions at more than $5 \sigma$ confidence, and thousands at $3 \sigma$. However, more than $75 \%$ of the astrophysical information is contained in the emission from unresolved sources. For this reason, some of the most important BLAST results emerge from a statistical analysis of the intensity fluctuations in the maps, rather than from a study of individually detected sources.

Information about the distribution of sources versus flux density (the differential source counts) is in the map pixel histogram. Patanchon et al. (2009) has successfully used this 
"P(d)" analysis to show that parametric models of the differential source counts can be constrained by the map pixel histogram at the three BLAST wavelengths, independently, and at flux densities fainter than could be probed with detected sources only. The retrieved counts fall more rapidly in flux than would be expected for a population of sources showing no evolution in luminosity or density. Recent studies with SPIRE are in good agreement with these early BLAST results, and have extended the source counts both at the faint and bright end of the distribution (Clements et al. 2010; Glenn et al. 2010).

Stacking analysis using positional information of sources selected at different wavelengths is a powerful tool to estimate the contribution that a given class of object has to the submillimetre background. Even in the case where the stacking catalog has a high surface density, with several sources for each BLAST beam, stacking provides an unbiased estimate of the associated flux, under the assumption that the catalog is not correlated on scales comparable with the beam.

By stacking the BLAST fluxes selected at the positions of the $24 \mu \mathrm{m}$ sources detected in deep MIPS surveys, Marsden et al. (2009) have shown that the retrieved CIB from 250 to $500 \mu \mathrm{m}$ is consistent with the FIRAS measurements. A similar result was obtained by Dole et al. (2006) at wavelengths shorter than the peak of the CIB, confirming that these measurements are consistent with $24 \mu \mathrm{m}$ selected sources generating the full CIB. Pascale et al. (2009) have studied the redshift distribution of the CIB, and have related it to the history of obscured star formation in the Universe, which shows a steep increase between $0<z<1$, confirming early results obtained from optical measurements (Lilly et al. 1996; Steidel et al. 1999).

In the 50 square degree survey of the Vela Molecular Ridge, Netterfield et al. (2009) detect more than 1000 compact sources in a range of evolutionary stages. The massdependent life-time is found to be longer than has been found in previous surveys of either low or high-mass cores, and significantly longer than free-fall or likely turbulent decay times. This implies some form of non-thermal support for cold cores during the early stage of star formation, which could be provided by the magnetic field.

\section{Probing the roles of magnetic fields in star formation with BLASTPol}

One of the key goals of modern astrophysics is to understand the details of star formation: how their masses are determined, and what the dominant physical processes that regulate the overall rate of star formation are. Significant progress has been made on these questions in recent years. For example, observations of dust emission and extinction (Nutter \& Ward-Thompson 2007) show that the overall distribution of core masses mimics the distribution of stellar masses. Recent Herschel observations have shown that molecular clouds present a ubiquitous filamentary structure, in which long thin filaments form first, and then fragment into pre-stellar cores (André et al. 2010; Hill et al. 2011). However, fundamental questions regarding molecular cloud structure and star formation are still being debated (McKee \& Ostriker 2007). For example, some investigators argue that molecular clouds, as well as cores, clumps and filaments inside the clouds, are dynamic structures, whose lifetimes are approximately equal to their turbulent crossing times (Vázquez-Semadeni et al. 2006). Others favor longer lifetimes, of order several crossing times (Netterfield et al. 2009; Blitz et al. 2007; Goldsmith et al. 2008). If clouds and their sub-structures do indeed live longer than a crossing time, they require support against gravity. This support could be provided by magnetic fields, which in many 


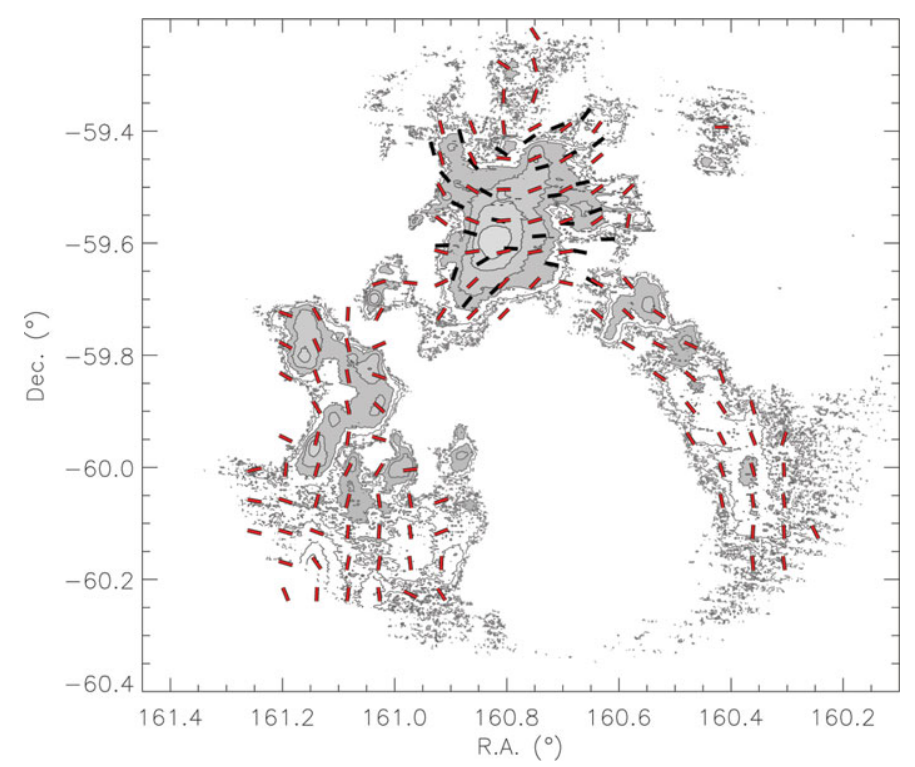

Figure 2. Preliminary results from the BLASTPol 2010 fight. The lines indicate the inferred magnetic field direction. Here we show a map made from 3 hours of BLASTPol data on the Carina Nebula. Total intensity contour levels measured at $500 \mu \mathrm{m}$ are shown in the background. The red pseudo-vectors are BLASTPol measurements. The black pseudo-vectors are measurements from the SPARO polarimeter at $450 \mu \mathrm{m}$ ( $\mathrm{Li}$ et al. 2006) showing a good agreement between the two detections.

numerical simulations dramatically affect both the star formation efficiency and the lifetime of molecular clouds (Li et al. 2010; Hennebelle et al. 2011). However, observationally, the strength and morphology of magnetic fields in molecular clouds have been poorly constrained. Zeeman splitting detections are limited to the brightest Galactic sources (Crutcher et al. 2010; Falgarone et al. 2008) and optical polarimetry is not possible in these regions of high extinction. The best method for probing these fields is far-IR and submillimeter polarimetry (Hildebrand et al. 2000; Ward-Thompson et al. 2000, 2009), where the radiation from asymmetric dust grains, aligned by the local magnetic field, is detected in polarization. BLASTPol is the first submillimeter polarimeter with both sufficient mapping speed to trace fields across entire clouds and sub-arcminute spatial resolution to trace the field at the scale of dense cores. This provides a critical link between the Planck all-sky polarization maps (with $5^{\prime}$ resolution) and the planned ALMA polarization measurements at ultra-high resolution of small individual sources (though with only a $20^{\prime \prime}$ field of view). BLASTPol data allow the first comprehensive detailed comparisons between observed magnetic fields in molecular clouds, and models derived from numerical simulations (Ostriker et al. 2001). Recent observations show that the extended sub-millimeter emission from molecular clouds is indeed polarized (Ward-Thompson et al. 2000, 2009; Li et al. 2006) and BLASTPol data is in good agreement with some of these early results (see next Section).

BLASTPol observations target the following three key questions in star formation and are discussed by Fissel et al. (2010): i) Is core morphology and evolution determined by large-scale magnetic fields? ii) Does filamentary structure have a magnetic origin? iii) What is the field strength, and how does it vary from cloud to cloud? 
Table 1. BLASTPol 2010 Observed Targets

\begin{tabular}{|l|c|c|c|}
\hline Target & $\begin{array}{c}\text { Distance } \\
(\mathrm{pc})\end{array}$ & $\begin{array}{c}\text { Obs. Time } \\
\text { (hours) }\end{array}$ & No. of B-vectors, 2010 \\
\hline Vela C & $\sim 700$ & 64 & $\sim 250$ \\
Lupus I & $\sim 200$ & 62 & $\sim 40$ \\
Puppis Cloud Complex & $\sim 1,000$ & 22 & TBD \\
IRDC filaments & $\sim 2,000-4,000$ & 8 & $T B D$ \\
cool GMCs & $\sim 3,000-5,000$ & 18 & TBD \\
Carina Nebula & $\sim 3,000$ & 3 & $\sim 100$ \\
\hline
\end{tabular}

\section{BLASTPol Observations}

During our initial fight of BLASTPol in 2010, most components worked flawlessly during the entire 9 day Antarctic flight and we were able to obtain degree-scale polarization maps of several nearby molecular clouds. The quality of the data were unfortunately limited by the failure of one of our spectral blocking filters. The filter was damaged just before launch or during ascent, and the result was a loss of angular resolution as well as a high instrumental polarization $(\sim 5 \%)$ that varies across the focal plane. In spite of this problem, analysis of four sources has been successfully completed: Vela C, Lupus I, and our bright calibrators Carina Nebula (see Figure 2) and G331.5-0.1. For the latter two targets our results are consistent with those obtained using SPARO at South Pole station (Li et al. 2006). In Vela C (Fissel et al., in preparation) and Lupus I (Matthews et al., in preparation), we detect coherent polarization at the level of a few percent for most sightlines, providing valuable new information on the large-scale polarization properties of these nearby star-forming regions. A summary of the full target list for the 2010 flight is given in Table 1 (See also http://blastexperiment.info). A plan has been implemented to better protect the blocking filter that failed in 2010, and we are now preparing for a second BLASTPol flight, planned for December 2012 - January 2013. During this second flight we expect to map more sky area, with much higher angular resolution and better sensitivity, which will lead to an increase in the number of vectors per target cloud by a factor of at least 10 compared to the 2010 observations.

\section{Acknowledgements}

The BLAST collaboration acknowledges the support of NASA through grant numbers NAG5-12785, NAG5-13301 and NNGO-6GI11G, the Canadian Space Agency (CSA), the Leverhulme Trust through the Research Project Grant F/00 407/BN, Canada's Natural Sciences and Engineering Research Council (NSERC), the Canada Foundation for Innovation, the Ontario Innovation Trust, the Puerto Rico Space Grant Consortium, the Fondo Istitucional para la Investigacion of the University of Puerto Rico, and the National Science Foundation Office of Polar Programs; C. B. Netterfield also acknowledges support from the Canadian Institute for Advanced Research. We would also like to thank the Columbia Scientific Balloon Facility (CSBF) staff for their outstanding work.

\section{References}

André, P., Men'shchikov, A., Bontemps, S., et al. 2010, A\&A, 518, L102

Blitz, L., Fukui, Y., Kawamura, A., et al. 2007, in Protostars and Planets V, ed. B. Reipurth, D. Jewitt, \& K. Keil, 81-96

Chandrasekhar, S. \& Fermi, E. 1953, ApJ, 118, 113

Chattopadhyay, G., Glenn, J., Bock, J. J., et al. 2003, IEEE Trans. Micro. T. Tech 
Clements, D. L., Rigby, E., Maddox, S., et al. 2010, A\&A, 518, L8

Crutcher, R. M., Wandelt, B., Heiles, C., Falgarone, E., \& Troland, T. H. 2010, ApJ, 725, 466

Devlin, M. J., Ade, P. A. R., Aretxaga, I., et al. 2009, Nature, 458, 737

Dole, H., Lagache, G., Puget, J.-L., et al. 2006, A $E A$, 451, 417

Falgarone, E., Troland, T. H., Crutcher, R. M., \& Paubert, G. 2008, A\& A, 487, 247

Fissel, L. M., Ade, P. A. R., Angilè, F. E., et al. 2010, in Society of Photo-Optical Instrumentation Engineers (SPIE) Conference Series, Vol. 7741

Glenn, J., Bock, J. J., Chattopadhyay, G., et al. 1998, in Proc. SPIE, Advanced Technology MMW, Radio, and Terahertz Telescopes, Thomas G. Phillips; Ed., Vol. 3357, 326-334

Glenn, J., Conley, A., Béthermin, M., et al. 2010, MNRAS, 409, 109

Goldsmith, P. F., Heyer, M., Narayanan, G., et al. 2008, ArXiv e-prints, 802

Griffin, M. J., Abergel, A., Abreu, A., et al. 2010, A\&A, 518, L3

Hennebelle, P., Commerçon, B., Joos, M., et al. 2011, A\&A, 528, A72

Hildebrand, R. H., Davidson, J. A., Dotson, J. L., et al. 2000, PASP, 112, 1215

Hill, T., Motte, F., Didelon, P., et al. 2011, A\& $A$, 533, A94

Li, H., Griffin, G. S., Krejny, M., et al. 2006, ApJ, 648, 340

Li, Z.-Y., Wang, P., Abel, T., \& Nakamura, F. 2010, ApJ, 720, L26

Lilly, S. J., Le Fevre, O., Hammer, F., \& Crampton, D. 1996, ApJ, 460, L1+

Marsden, G., Ade, P. A. R., Benton, S., et al. 2008, in Society of Photo-Optical Instrumentation Engineers (SPIE) Conference Series, Vol. 7020

Marsden, G., Ade, P. A. R., Bock, J. J., et al. 2009, ApJ, 707, 1729

McKee, C. F. \& Ostriker, E. C. 2007, ARA\& $A, 45,565$

Moncelsi, L., Ade, P., Elio Angile, F., et al. 2012, ArXiv astro-ph: 1208.4866

Netterfield, C. B., Ade, P. A. R., Bock, J. J., et al. 2009, ApJ, 707, 1824

Nutter, D. \& Ward-Thompson, D. 2007, MNRAS, 374, 1413

Ostriker, C., Stone, J. M., \& Gammie, C. F. 2001, ApJ, 546, 980

Pascale, E., Ade, P. A. R., Bock, J. J., et al. 2008, ApJ, 681, 400

Pascale, E., Ade, P. A. R., Bock, J. J., et al. 2009, ApJ, 707, 1740

Patanchon, G., Ade, P. A. R., Bock, J. J., et al. 2009, ApJ, 707, 1750

Steidel, C. C., Adelberger, K. L., Giavalisco, M., Dickinson, M., \& Pettini, M. 1999, ApJ, 519,1

Valiante, E., Ade, P. A. R., Bock, J. J., et al. 2010, ApJS, 191, 222

Vázquez-Semadeni, E., Ryu, D., Passot, T., González, R. F., \& Gazol, A. 2006, ApJ, 643, 245

Viero, M. P., Ade, P. A. R., Bock, J. J., et al. 2009, ApJ, 707, 1766

Ward-Thompson, D., Kirk, J. M., Crutcher, R. M., et al. 2000, ApJ, 537, L135

Ward-Thompson, D., Sen, A. K., Kirk, J. M., \& Nutter, D. 2009, MNRAS, 398, 394 